\title{
PRUEBAS ESTANDARIZADAS PARA EL ACCESO A LA EDUCACIÓN SUPERIOR EN CHILE: PERFORMATIVIDAD Y SUBJETIVIDAD DE LOS ESTUDIANTES $^{1}$
}

\section{Francisco Durán del Fierro ${ }^{2}$}

\begin{abstract}
RESUMEN
El presente estudio tiene como objetivo identificar las principales diferencias y semejanzas en la subjetividad de los estudiantes que finalizan la educación secundaria en relación con las pruebas de competencias, desarrolladas por el Departamento de Evaluación, Medición y Registro Educacional de la Universidad de Chile (DEMRE) como propuesta para el nuevo sistema de admisión, en comparación con la Prueba de Selección Universitaria (PSU). Mediante entrevistas semiestructuradas a una muestra de estudiantes que participaron en el primer piloto de las nuevas pruebas (2017), y que además estaban inscritos para rendir la PSU (proceso 2018), se encontró que estos valoraron positivamente la propuesta del DEMRE, puesto que avanza en pertinencia — el contenido de lo evaluado- y equidad -igualdad de oportunidades_- Sin embargo, los estudiantes entrevistados de la clase baja manifestaron que estas pruebas podrían producir nuevas formas de segregación social, más sutiles y soterradas y, por tanto, ser potencialmente más injustas que la PSU. Para comprender en mayor profundidad la forma en que las nuevas pruebas afectan - performatividad- la subjetividad de los estudiantes, es fundamental considerar los diferentes contextos en que estas se pudieran aplicar.
\end{abstract}

Conceptos clave: enseñanza secundaria, enseñanza superior, performatividad, subjetividad, test de rendimiento.

\section{STANDARDIZED ADMISSION TESTS TO ACCESS HIGHER EDUCATION IN CHILE: PERFORMATIVITY AND STUDENTS' SUBJECTIVITY}

\begin{abstract}
This study aims to identify the main differences and similarities of students' subjectivity regarding the new skills' assessment tools, developed by the Department of Educational Evaluation, Assessment and Registry at the University of Chile (DEMRE) for the new admissions system, in lieu of the current University Selection Test (PSU, for its acronym in Spanish). Drawing on semistructured interviews carried out with a sample of students, who participated in the first pilot
\end{abstract}

$1 \quad$ Este estudio es parte del proyecto Fondef iD16110090 "Habilidades y competencias para la educación superior. Desarrollo de instrumentos y propuestas que aporten a un nuevo sistema de admisión" (2017-2019) financiado por Conicyt.

2 Departamento de Evaluación, Medición y Registro Educacional (DEMRE), Universidad de Chile, Santiago. Chile. Contacto: fdurandelfierro@u.uchile.cl 
study (2017) and were also registered in the latest PSU admission process (2018), the findings show that students recognize DEMRE's proposal as positive on two counts; it is more relevant (regarding the assessed content) and fairer (equal opportunities). However, students from lower classes argue that these new assessment tools have the potential to produce new, less conspicuous forms of social segregation and, therefore, could possibly be less fair than the current test (PSU). Further research is needed to understand in greater depth the way in which the new assessment tools affect -performativity- students' subjectivity in relation to social classes, particularly in the context of the implementation of these new assessment tools.

Key concepts: achievement test, higher education, performativity, secondary education, subjectivity.

\section{Introducción}

\section{Educación superior y procesos de admisión}

El sistema de educación superior (en adelante ES) chileno es masivo, diverso y socialmente estratificado (Mineduc, 2016; Orellana, 2011; Torres y Zenteno, 2011). Por una parte, los estudiantes de familias de altos ingresos se concentran en las universidades tradicionales y en las nuevas universidades privadas de perfil religioso o empresarial. Todas ellas son selectivas en términos académicos (altos puntajes en la Prueba de Selección Universitaria, en adelante, PSU) o económicos (altos aranceles). Por otra parte, los estudiantes de familias de bajos ingresos se ubican principalmente en las universidades privadas masivas, institutos profesionales y centros de formación técnica, los que no utilizan la PSU como instrumento de selección. A nivel de carreras ocurre un fenómeno similar. Los primeros se concentran en carreras de nivel profesional y licenciatura (Medicina, Derecho, Ingeniería Civil, entre otras), mientras los segundos lo hacen en carreras de nivel técnico o profesional sin licenciatura con una marcada división de género: los hombres en carreras tecnológicas y las mujeres en carreras vinculadas a la educación y salud (Orellana, 2011; Orellana, Guzmán, Bellei, Gareca y Torres, 2017).

En este contexto, la elección de una carrera o institución es un proceso situado socialmente. Es decir, no se trataría de una elección racional que depende de la concurrencia de información oportuna y eficaz. Más bien, implica la movilización de distintas estrategias 
condicionadas por la posición social del individuo (Bourdieu \& Passeron, 1990). Esta condición, que subyace al momento decisional, implica que la experiencia que tienen los sujetos con los instrumentos de evaluación para el acceso a la ES sea distinta según dicha posición, incluso antes de enfrentarse directamente a ellos. Es decir, es disímil la mirada y visión de un sujeto al que le resulta ajena y distante una prueba estandarizada y competitiva, respecto de otro que ha sido formado y se ha preparado para ella. No solo se trata de la capacidad efectiva de rendirla, sino también de la autoexclusión que ocurre por la sola existencia de la prueba y la experiencia de lo deseable y lo posible que le subyace.

El principal instrumento que regula el acceso a las universidades selectivas es la PSU. Si bien se han introducido distintos mecanismos de racionalización para la elección de la carrera e institución (acreditación institucional y de carreras, información sobre retornos e ingresos, la plataforma Elige carrera ${ }^{3}$, rankings, entre otros), esta prueba ocupa quizás el papel más importante en este contexto. La PSU determina cómo se distribuyen los estudiantes en las distintas instituciones y carreras del sistema; sin embargo, solo $39^{4}$ de 61 universidades del país utilizan sus resultados para seleccionar a sus alumnos. En consecuencia, un grupo importante de estudiantes ingresa a carreras e instituciones no selectivas ${ }^{5}$, produciéndose un cisma que no solo se refleja en la selección, sino también durante un momento previo y fundamental: cuando se imagina-proyecta el futuro en la educación superior.

En este panorama, diverso y socialmente complejo, es posible sostener que la selección universitaria y los instrumentos de evaluación que la acompañan influyen en el proceso de toma de decisión que realizan los estudiantes al trazar su camino hacia la educación superior. Asimismo, su presencia produce y reconfigura las

3 Página web que ofrece un conjunto de evaluaciones (de intereses y habilidades) para tomar una decisión informada (eligecarrera.cl). Esta plataforma fue desarrollada por el Consejo Nacional de Educación (CNED).

4 En el proceso de admisión 2018.

5 Si se consideran como no selectivas aquellas instituciones que no están adscritas al Sistema Único de Admisión (SUA), entonces aproximadamente el 53\% de estudiantes se encuentra matriculado en este tipo de instituciones. 
percepciones, valoraciones y expectativas de los estudiantes, incluso entre aquellos que no pretenden ingresar a instituciones selectivas. En definitiva, la existencia de estas pruebas tiene efectos en la realidad social de todos los estudiantes que finalizan la educación media, no solo en aquellos que están decididos a rendirlas.

Así, el proceso de elección de los estudiantes se ve influenciado por factores externos, donde la modificación de uno de ellos implica la reconfiguración de la subjetividad involucrada, y la lógica electiva que le da sentido. Por ejemplo, si en la década de los noventa las significaciones sociales —o narrativas culturales- que predominaban entre los jóvenes chilenos giraban entre la resistencia contestataria y la apatía hacia los asuntos públicos, lo que configura a las actuales narrativas de los estudiantes es mucho más heterogéneo y abarca desde la preocupación por el endeudamiento — posición de consumidorhasta la movilización activa por los temas de interés público, como la crítica a la mercantilización del sistema (Canales, Ghiardo, \& Opazo, 2015; Canales, Opazo, \& Camps, 2016). Por lo tanto, la modificación de un factor del proceso de admisión —en este caso, la prueba estandarizada - tiene consecuencias en las percepciones, deseos, valoraciones, expectativas y modos de entendimiento de los estudiantes que finalizan la educación secundaria, y en el sentido que le atribuyen cuando se constituyen las estrategias y acciones para ingresar a la educación superior.

\section{Dispositivos de evaluación y subjetividad}

La preocupación por los procesos de subjetivación ha sido abordada desde diferentes enfoques teóricos. Por una parte, se ha tratado de indagar acerca de cómo ciertos dispositivos gubernamentales afectan la subjetividad de los sujetos. Aquí es posible encontrar una extensa reflexión teórica e investigación empírica cuyo objetivo ha sido analizar críticamente esta relación. Por otra parte, es posible encontrar toda una corriente psicológica y sociológica, también con ingentes estudios empíricos, que buscan mostrar cómo se forman las aspiraciones, percepciones y valoraciones de los estudiantes al momento previo de ingresar a la educación superior. En esta misma línea se ubica la economía de la educación, que ha desarrollado un 
programa de investigación estrechamente vinculado con el diseño de políticas educativas. A pesar de la diversidad de enfoques posibles para analizar los procesos de subjetivación, este artículo propone —al menos desde un punto de vista general —, una interpretación basada en una posición postestructuralista (Peters \& Burbules, 2004).

En este sentido, la premisa inicial dice relación con sostener que en las sociedades modernas existe una diversidad de dispositivos de poder que producen y afectan la totalidad de la experiencia de los sujetos, directa o indirectamente. Según Ramos (2012), a partir de las ideas de Foucault (1982; 2009; 2010a; 2010b), las tecnologías de poder, dependientes de los dispositivos gubernamentales (Dean, 2010; Rose, 2005), tienen capacidad performativa en la subjetividad de los sujetos, así como también en las prácticas institucionales (Callon, 2007;2010; MacKenzie, Muniesa \& Siu, 2008). Es decir, una tecnología — como podría ser una prueba estandarizada — no solo es un dispositivo que cumple con objetivos procedimentales ${ }^{6}$, sino también es productor de realidades que modifican —espacial y temporalmente- las estructuras de sentimientos y de prácticas de los sujetos, epistemológica y ontológicamente. Lo que se ve afectado es tanto la acción como el pensar "íntimo" de los sujetos (Ball, 2003, 2015; Ball \& Olmedo, 2013).

Por lo tanto, es posible sostener que la subjetividad de los estudiantes se ve continuamente modificada por los distintos dispositivos de poder, cuya profundidad y carácter depende del tipo de tecnología que interviene y la forma en que aparece o se ausenta. A partir de este enfoque teórico, la PSU no es sino una tecnología político-moral con repercusiones en la realidad individual y social de los estudiantes, tanto de aquellos que la rinden como de los que no lo hacen. Asimismo, tiene efectos en las instituciones, particularmente en los procesos de enseñanza-aprendizaje, tanto en la educación escolar como en la educación superior: en el primer caso, redefine las formas de enseñar y de aprender, enfatizando en la preparación para el test; en el segundo, influye en el diseño de los programas de nivelación y seguimiento, haciéndolos equivalentes a los resultados

Por ejemplo, evaluar y predecir el desempeño de los estudiantes. 
de la prueba ${ }^{7}$. Así, los dispositivos de evaluación modifican e inciden en las percepciones, deseos, valoraciones y expectativas de los sujetos, como también en las prácticas institucionales (Ball, 2017).

Sin embargo, ¿de qué se habla cuando se hace referencia a la "subjetividad" de los estudiantes? En términos generales, las filosofías del sujeto han entendido a la subjetividad como el fundamento del pensamiento y las prácticas sociales (racionalismo) o, también, como aquel lugar de la subyugación o de la manipulación (Zima, 2015). En específico, por una parte se trata del lugar donde se forma la autoimagen, el sentido del ser y de los otros y la posibilidad de existencia en relación con ciertas estructuras de emociones, sentimientos y memorias (Ball, 2003; Burrows, 2012) y, por otra, es el lugar de resistencia contra las formas de poder que actúan sobre el sujeto (Foucault, 1982). La subjetividad, en este sentido, es el espacio de contacto, de articulación, entre el ser y el poder, pero también donde ocurren las luchas que permiten darle sentido a los procesos sociales (Ball, 2017).

Algunos autores incluso han indicado que las sociedades modernas son sociedades performativas, al estilo de "fabricaciones" de sujetos (Ball, 2003). En educación, la performatividad refiere a una tecnología, a un modo de regulación que emplea juicios y comparaciones que tienen consecuencias, en términos de premios y sanciones, tanto materiales como simbólicas en los sujetos que participan (Ball, 2003, 2012, 2017; Foucault, 1982; 2010a; 2010b). Así, la performatividad no apunta a modificar las percepciones, valoraciones y prácticas de los individuos en virtud de lo que es correcto o más apropiado, aunque es posible una crítica desde esa perspectiva. El principal propósito es más bien cambiar lo que los sujetos son como tal. Por tanto, se trata de un asunto que ocurre en el plano ontológico. Con ello, lo que surge son diferentes tipos de sujetos que, en función de las relaciones que lo constituyen, conducen sus prácticas y modos de ser.

Por ejemplo, los programas de nivelación que ofrecen las universidades suelen diseñarse en función de las materias y resultados de la PSU, dejando de lado el desarrollo de competencias y habilidades que, incluso, son escasas en estudiantes con buenos puntajes. 
Sin embargo, en los estudios postestructuralistas la subjetividad suele enfocarse en las narrativas o en el testimonio de los sujetos (identidades), principalmente desde las diferencias de género, étnicas o sexuales. En cierta medida, este enfoque resulta problemático cuando se investiga en espacios sociales altamente segmentados en términos socioeconómicos. Por tal razón, se han esbozado algunas críticas a la idea de identidad como mero testimonio, desligada de las relaciones sociales que la producen (Neary \& Winn, 2016; Sutton, 2017). En ese sentido, al analizar la subjetividad es necesario entonces apuntar hacia una interpretación que permita balancear ambas aproximaciones y entender la subjetividad como expresiones narrativas situadas socialmente.

En definitiva, y en función de lo anteriormente planteado, es posible sostener que la PSU, como dispositivo de evaluación, regula los premios y sanciones sociales de los estudiantes. Así, por ejemplo, se manifiestan sentimientos de culpa y vergüenza, así como también de orgullo y esfuerzo que perduran incluso a lo largo de la vida profesional. La subjetividad de los estudiantes queda trastocada por la experiencia de éxito o fracaso. Asimismo, la relación que los estudiantes tienen con la PSU no solo se produce en términos del conocimiento involucrado, es decir, en relación con la materia que deben dominar para conseguir un resultado, sino también entre los mismos estudiantes — que se convierten en competidores por vacantes- y con las instituciones. Por tanto, es un dispositivo que, de manera permanente, reconfigura la totalidad de las relaciones: entre los estudiantes con el conocimiento, con el otro y con las formas de organización social.

En cuanto al sentido normativo que los individuos le atribuyen a ciertas políticas e instrumentos, es necesario considerar algunas aproximaciones conceptuales relacionadas con lo que la literatura ha denominado "justicia social" (Dubet, 2012). En ocasiones, hay desigualdades que son concebidas como injustas puesto que no cumplen con ciertos requerimientos. La forma de legitimar estas desigualdades es generando las condiciones que permitan que cada individuo tenga las mismas oportunidades de situarse en ciertas posiciones sociales. Esto es lo que habitualmente se denomina "justicia procedimental". Sin embargo, también es posible evaluar 
las desigualdades en función de la "justicia distributiva", la cual enfatiza en la distribución de los recursos que reciben los individuos en comparación con el resto.

Por tanto, la justicia procedimental considera injustas aquellas situaciones en que no se cumple con los estándares procesuales mínimos reconocidos como legítimos en un momento determinado. La preocupación, por tanto, no es tanto por las desigualdades que se producen, sino por asegurar las condiciones que permitan que cada individuo tenga las mismas oportunidades de situarse en ciertas posiciones sociales, a pesar de los resultados posteriores (Marshall, 1973; Rawls, 2005). En este caso, las diferencias en los resultados de las pruebas estandarizadas serían justas en la medida en que el instrumento no discrimine a ningún grupo social. Por ejemplo, un instrumento de evaluación podría considerarse injusto si su diseño pone en situación de desventaja a un grupo específico de estudiantes.

Por su parte, la justicia distributiva apela a una distribución equitativa de los recursos que una sociedad dispone, a fin de disminuir la distancia al interior de la estructura social (Barozet y Mac-Clure, 2015; Turner, 2007). Así, los resultados en las pruebas de acceso a la educación superior serían injustos ya que reproducen condiciones previas que no han sido soslayadas, independientemente de las condiciones de igualdad - y confiabilidad ${ }^{8}$ - que contemple el instrumento.

\section{Pruebas de competencias para el acceso a la educación superior}

En 2017, el DEMRE de la Universidad de Chile 9 inició un proyecto de investigación ${ }^{10}$ con el propósito de desarrollar nuevos instrumentos de evaluación (Pruebas de Competencias, PC en adelante) adecuados a la diversidad de instituciones, programas y postulantes a la educación superior para aportar al nuevo sistema de admisión propuesto

3 Entendida, según la psicometría, como la precisión y estabilidad de una medición.

9 Organismo encargado de diseñar las pruebas de selección a las universidades adscritas al Sistema Único de Admisión.

10 Proyecto Fondef (2017-2019) "Habilidades y competencias para la educación superior. Desarrollo de instrumentos y propuestas que aporten a un nuevo sistema de admisión". 
inicialmente por el proyecto de ley de reforma a la educación superior $y$, posteriormente, ratificado por la Ley $\mathrm{N}^{\circ}$ 21.091. El objetivo de estas pruebas ${ }^{11}$ es evaluar competencias fundamentales y habilidades transversales necesarias para cursar exitosamente un programa de estudios en ES, tanto para el subsistema técnico como para el profesional $^{12}$. En 2017, se diseñaron las primeras pruebas y se aplicó un piloto a 6.392 estudiantes de cuarto año medio de las distintas modalidades educativas y tipos de establecimiento en cuatro regiones del país. En 2018 se realizó el segundo piloto con la participación de aproximadamente 2.500 estudiantes de cuarto año medio.

En este contexto, resulta relevante conocer las diferencias y semejanzas que devienen en la subjetividad de los estudiantes frente a una nueva batería de instrumentos, considerando sus distintos orígenes sociales. Los estudiantes son los actores principales del proceso de admisión, cuestión que motiva comprender las percepciones y valoraciones que le atribuyen. Adicionalmente, se constatan escasos estudios que exploren cómo las políticas de educación reconfiguran las subjetividades y narrativas de los estudiantes. Tampoco se observan análisis que permitan comprender las valoraciones normativas que los estudiantes despliegan respecto de los instrumentos de selección a la ES. Esta investigación se sitúa en el intento de aportar evidencia acerca de ambas cuestiones, tanto de la PSU como de las nuevas PC.

\section{Objetivo y preguntas de investigación}

El presente estudio busca identificar las principales diferencias y semejanzas en la subjetividad de los estudiantes que finalizan la educación secundaria en relación con las pruebas estandarizadas

11 Estas pruebas miden competencias en las áreas de Matemática, Lectura, Escritura y Ciencias y, además, de un cuestionario de habilidades transversales. Puesto que se busca medir competencias más que contenidos curriculares, durante el proyecto se han probado distintos tipos de ítems: selección múltiple, respuesta breve y respuesta abierta, entre otros.

12 Esto no implica que sean las únicas pruebas. El proyecto busca diseñar aquellas pruebas que sean comunes para los estudiantes provenientes de todas las modalidades educativas de la educación secundaria y, además, pertinentes para todas las IES del sistema. complementariamente a estas pruebas comunes, se deberían diseñar e implementar otras pruebas más específicas acordes con las necesidades particulares de algunas instituciones o áreas disciplinares. 
(PSU y las PC) en el contexto del acceso a la ES. De esa manera, las preguntas de investigación son las siguientes:

- ¿Cuáles son las percepciones y valoraciones que articulan los estudiantes en relación con las pruebas estandarizadas diseñadas para ingresar a la ES? ¿Cuáles son las principales diferencias y semejanzas entre la PSU y las PC?

- ¿En qué medida estas percepciones y valoraciones difieren según origen social o el tipo establecimiento al que asisten?

\section{Metodología}

Las PC se encuentran en proceso de diseño y ajuste desde el año 2017 a la fecha. En julio y agosto de 2017 se realizó el primer pilotaje a estudiantes de cuarto año medio en cuatro regiones del país (Metropolitana, Coquimbo, O’Higgins y Biobío), pertenecientes a distintas modalidades educativas $\left(\mathrm{HC}-\mathrm{TP}^{13}\right)$ y dependencia administrativa (PP-PS-MU ${ }^{14}$ ).

En este sentido, se trata de una investigación exploratoria que busca indagar en las percepciones y valoraciones de los estudiantes en relación con las nuevas PC en comparación con la PSU ${ }^{15}$. En particular, resulta relevante indagar en las percepciones y valoraciones de los estudiantes provenientes de la modalidad TP, puesto que el diseño de la $\mathrm{PSU}^{16}$ no reconoce adecuadamente su trayectoria formativa ${ }^{17}$.

\subsection{Producción y análisis de información}

La producción de información se realizó a través de entrevistas semiestructuradas que se aplicaron a una muestra de estudiantes participantes del primer piloto de las PC e inscritos para rendir

13 Humanista científica y técnico profesional.

14 Particular pagado; particular subvencionado; y municipal.

15 Se trata de explorar solo en estos aspectos de la "subjetividad" la que, como se señaló en el marco teórico, contempla otras variables.

16 La construcción de ítems de la PSU se basa en los contenidos mínimos obligatorios del currículo HC.

17 De hecho, la brecha de resultados entre TP y HC en la PSU es mayor a una desviación estándar, que es valor alto en estos términos (Lavados et al., 2018). 
la PSU admisión 2018. Para analizar la información, se aplicó el análisis de contenido a partir de categorías previamente definidas. Es decir, se trató de una aproximación deductiva a partir de la revisión teórica presentada y ajustada en virtud de los datos construidos. Con ello, se identificaron los principales discursos en relación con las preguntas y objetivos de investigación. Para indagar en las diferencias sociales, en ambos casos —PSU y PC—, se realizó un análisis teniendo en cuenta la variable clase social, distinguiendo entre clase alta y clase baja, dada la centralidad que juega en la conducta de los estudiantes en educación (Bourdieu \& Passeron, 1990; Orellana et al., 2017).

\subsection{Muestra}

Para las entrevistas se seleccionaron estudiantes de cuarto año medio que participaron en el piloto del 2017, los que a su vez estaban inscritos para rendir la PSU para el proceso 2018. Lo anterior se realizó basado en una muestra intencionada siguiendo el principio de variación estructural ${ }^{18}$. Para su validación, la muestra siguió el criterio de saturación, es decir, consideró un proceso acumulativo, secuencial y flexible en la inclusión de nuevos casos.

Para la selección de estudiantes se definieron tres criterios: tipo de establecimiento (dependencia), modalidad y grupo social. Además, se consideraron todas las regiones en las que se aplicó el piloto y se resguardó la representación de género.

Para determinar el origen social de los estudiantes se definieron los siguientes criterios:

- porcentaje de padres del establecimiento que pertenecen a la primera categoría de la estructura ocupacional (según lo señalado en el formulario de inscripción a la PSU proceso admisión 2017);

- grupo socioeconómico del establecimiento educacional, según indicador Simce ${ }^{19}$;

18 Se seleccionan individuos según las distintas posiciones en las que se ubican en una estructura social determinada, identificando grupos representativos de cada categoría. Lo que se busca es caracterizar cómo los estudiantes reconstruyen su discurso desde una posición social.

19 Índice construido a partir de variables de ingreso de los padres del colegio respectivo. 
- promedio puntaje PSU Lenguaje y Matemática del establecimiento educacional.

Con estas variables se definieron los establecimientos que mejor representaban a ciertos grupos sociales, divididos entre clase alta y clase baja. La principal premisa fue que asistir a uno de estos establecimientos —homogeneidad interna en términos socioeconómicos - tiene implicancias en la constitución de las subjetividades de los estudiantes.

\subsection{Algunas consideraciones metodológicas}

Los estudios que analizan las clases sociales buscan identificar algunos elementos comunes que le dan sentido a la pertenencia a esos espacios sociales, a pesar de la heterogeneidad interna que las caracteriza. En este estudio, se asume la dificultad de usar estos conceptos y el propósito no es simplificar la diversidad de subjetividades que es posible encontrar, ni generalizar las percepciones y valoraciones por clase. Al contrario, la premisa fundamental es que hay ciertos patrones comunes que es posible analizar y, a la vez, contradicciones y fragmentaciones internas en la subjetividad (Barnett \& Di Napoli, 2008).

En cuanto a la muestra final, por motivos de tiempo y recursos no fue posible saturar la percepción y valoraciones de la clase alta. Por tanto, los resultados que se muestran son provisorios y requieren de un análisis más profundo y cuidadoso de las distintas percepciones y valoraciones que se construyen allí. Sin embargo, como han señalado estudios recientes enfocados en este sector social, es posible identificar una cierta "aura" o distinciones en la "elite", que el resto de la sociedad reconoce como tal, a pesar de no estar necesariamente cohesionada (Méndez \& Gayo, 2019). Por tanto, aun cuando no se cubren todas las opciones posibles, se asume que es posible captar algunos elementos que son comunes y que son reconocidos como tales por el resto de la sociedad.

En total, se entrevistó a 20 estudiantes, distribuidos de la siguiente manera ${ }^{20}$ :

20 En el pilotaje de 2017 participaron 6.392 estudiantes, de los cuales el 17\% era de la IV Región, el 12\% de la VI Región, el 19\% de la VIII Región y el 50\% de la Región Metropolitana. 
Tabla 1

Muestra de estudiantes entrevistas semiestructuradas

\begin{tabular}{lllcccc}
\hline & \multicolumn{3}{c}{ Región } & & & \\
\hline Dependencia & & IV & VI & VIII & RM & Total \\
& M & 1 & 1 & 2 & 2 & 6 \\
& PS & 2 & 2 & 1 & 4 & 9 \\
& PP & 1 & 1 & 1 & 2 & 5 \\
& Total & 4 & 4 & 4 & 8 & 20 \\
\hline Modalidad & & & & & & \\
& HC & 2 & 2 & 2 & 4 & 10 \\
& TP & 2 & 2 & 2 & 4 & 10 \\
& Total & 4 & 4 & 4 & 8 & 20 \\
\hline Clase social & Alta & 1 & 1 & 1 & 2 & 5 \\
& Baja & 3 & 3 & 3 & 6 & 15 \\
& Total & 4 & 4 & 4 & 8 & 20 \\
\hline Género & & & & & & \\
& Hombre & 0 & 2 & 1 & 5 & 8 \\
& Mujer & 4 & 2 & 3 & 3 & 12 \\
& Total & 4 & 4 & 4 & 8 & 20 \\
\hline
\end{tabular}

Fuente: Elaboración propia.

\section{Principales hallazgos}

\subsection{Performatividad de los instrumentos de evaluación y percepciones y valoraciones de los estudiantes}

La presencia de pruebas estandarizadas orientadas a la selección para la educación superior moldea la subjetividad de aquellos estudiantes — como individuos y grupo social— que cursan la enseñanza media. Esta presencia, espectral o material, lejana o cercana, influye en las significaciones que construyen. Por tanto, estos instrumentos tienen una capacidad "performativa", puesto que inciden en los imaginarios que edifican tanto los estudiantes como sus familias, en lo pensable y lo no pensable, en las proyecciones y expectativas que construyen y reconstruyen una y otra vez $z^{21}$.

21 Ciertamente, también hay otros factores iguales o más importantes que configuran su subjetividad en este contexto, como el costo de la carrera, la duración y la calidad, entre otros. 
Así, la existencia de pruebas cuyas consecuencias inciden en las trayectorias formativas y laborales de los individuos no solo tiene efectos prácticos y materiales, sino también da forma a los imaginarios sociales. Asimismo, estos imaginarios están presentes no solo en aquellos estudiantes decididos a rendir estas pruebas, sino también en los que renuncian a darlas, por diferentes motivos. En específico, se asume que condiciona tanto a los estudiantes de la modalidad HC como a aquellos de TP. Es decir, el hecho de que un estudiante decida seguir una carrera que no tiene como requisito rendir la prueba, no significa que, en algún momento decisional, su existencia no haya tenido un efecto sobre su decisión final. El carácter performativo de estos instrumentos radica precisamente en eso: redefine la totalidad de la realidad social en la cual se inserta, a sus agentes y las relaciones sociales que los constituyen.

El efecto performativo en un grupo de estudiantes es lo que se indaga en esta sección, tanto de la PSU como de las PC. Para efectos del análisis, se asume la subjetividad de los estudiantes como las "percepciones" y "valoraciones" que ellos construyen, cuyo basamento biográfico es social, dejando para otros estudios aspectos ligados a la subjetividad, como las prácticas o estrategias. Las percepciones se relacionan con aquellas opiniones y emociones que hacen referencia a los aspectos instrumentales de las pruebas, como la dificultad y los contenidos de los ítems. Las valoraciones refieren al sentido normativo que los sujetos le atribuyen a estos instrumentos, vinculados a un cierto ideario de justicia social.

\subsubsection{Prueba de Selección Universitaria (PSU)}

a. Elementos comunes ${ }^{22}$

A pesar de las distintas críticas que recibe, los estudiantes estiman que la PSU juega un papel vital en el proceso decisional para el ingreso a la educación superior. Es más, se evidencia, más allá del instrumento en cuestión, una valoración positiva del rol que cumple la selección, como lógica de ingreso a estudios superiores. La PSU, por tanto, es

22 En las citas se identifica a los estudiantes a partir de la modalidad de enseñanza (HC y TP), el tipo de establecimiento (PP, PS y M) y la región donde habitan (IV, VI, VIII, RM). 
la expresión procedimental y simbólica de dicha lógica, motivo por el cual su existencia no es cuestionada del todo ${ }^{23}$.

Esta valoración es plausible puesto que la PSU contiene una promesa: el resultado depende esencialmente del esfuerzo. Se trataría de una reproducción cultural basada en el desempeño, donde los ganadores — de cualquier estrato social— no interiorizan ningún tipo de culpa o cuestionamiento, porque se trataría de una competencia leal. En ese sentido, la PSU sería el esfuerzo histórico por erradicar los mecanismos adscriptivos de diferenciación social.

Por lo tanto, la presencia de la PSU resultaría fundamental para legitimar la idea de un sistema público de educación superior, cuyo basamento es la igualdad de oportunidades. El carácter público se expresa en su propósito más sentido: objetivar y racionalizar el ingreso a la educación superior, a costa de los privilegios. Así, los resultados de la prueba no son sino la distribución jerárquica de los estudiantes basada en ese pretendido esfuerzo.

En otros términos, la PSU refleja el paradigma meritocrático en el sistema de acceso a la educación superior, garantizando la imparcialidad de los procesos de admisión. Así, en general los estudiantes no cuestionan su existencia, sino más bien el contexto de desigualdad donde se inserta.

siento que la PSU no facilita la equidad, pero siempre está el esfuerzo de uno, o sea, uno siempre puede ir a la biblioteca y conseguir material, entonces considero que no es tanto de equidad porque siempre va haber más esfuerzo o no (HC-PS-VIII).

Por otra parte, los entrevistados señalan de manera transversal que en el caso de que la PSU ya no fuera parte del proceso de selección no cambiarían la carrera, pero sí la institución. Aquí se aprecia el alcance respecto de la construcción de expectativas de la PSU: hay

23 Por cierto, esta conclusión general debe entenderse en un contexto en que la PSU y la lógica de selección ya han ejercido su fuerza performativa. Por tanto, esta valoración positiva no implica la inexistencia de una potencial resistencia y crítica a su implementación y lógica normativa. 
claridad de que ella regula el acceso a las instituciones más prestigiosas. Lo que condiciona la PSU no es necesariamente la vocación profesional, sino el acceso a espacios sociales y culturales de privilegio.

más que nada, yo a la PSU no la veo como algo que me haga decidir la carrera que yo quiero estudiar (HC-PS-VI).

Si me va muy bien en la PSU probablemente elija una [universidad] con un requisito mayor ... no sé, las típicas, la $\mathrm{PUC}^{24}$ o la Usach ${ }^{25}$ que son como las universidades más mencionadas, que tienen un puntaje de corte mucho más alto y son más exigentes con respecto a la PSU (HC-PS-VIII).

A partir del crecimiento de instituciones privadas de distinto tamaño, algunas más masivas que otras, el sistema ofrece la oportunidad de estudiar prácticamente cualquier carrera, en las distintas áreas del conocimiento, sin necesidad de obtener un buen resultado en la PSU. De esta forma, la diferenciación y la búsqueda de prestigio radican más bien en la institución a la que se logra ingresar. Por lo tanto, la PSU influye principalmente en la elección de la institución, a pesar de la "autoeficacia" que asume el estudiante.

b. Percepciones y valoraciones estudiantes entrevistados clase alta Los estudiantes entrevistados de clase alta, a diferencia de sus pares de clase baja, consideran que la PSU es un instrumento limitado en cuanto a su capacidad para definir quiénes son aptos para ingresar a la educación superior, puesto que excluye a potenciales talentos ocultos. Es posible identificar dos posiciones que respaldan esta idea.

Por una parte, son enfáticos en señalar que es el capital cultural el principal factor de éxito en la PSU. Es decir, estos estudiantes tienen plena confianza en las experiencias de aprendizaje que han tenido, tanto en el colegio como en el hogar. Esto muestra que la base sobre la cual construyen sus imaginarios y expectativas es de una relativa certidumbre. Se trata de una certeza que proviene de la posición

Pontificia Universidad Católica de Chile.

25 Universidad de Santiago de Chile. 
social en la que se encuentran, a diferencia de los que pertenecen a la zona baja de la distribución del ingreso, donde la incertidumbre es principalmente lo que moldea su subjetividad, como se verá más adelante. Esta confianza se aprecia en la siguiente afirmación:

yo creo que todo lo que entra en la prueba lo vimos en el colegio en una, en algún año, no hay nada que haya visto en preu externo que no haya visto en el colegio (HC-PP-VI).

En otras palabras, los estudiantes entrevistados de clase alta consideran que es posible tener un buen desempeño en la prueba mediante el conocimiento adquirido a través del capital cultural, esto es, la educación proveniente del hogar y la educación escolar. El aprendizaje emanado del primer espacio, según estos estudiantes, influye directamente en los resultados de la PSU. Así, por ejemplo, elementos como el fomento a la lectura se vuelven claves para un buen rendimiento en la PSU Lenguaje.

igual, si te inculcan la lectura, desde más chica hace la diferencia, pero va más por si tú lees, si te estás dando el tiempo de comprender lo que lees, si lees muy apurado, si tu familia lee, es muy importante. Cuántas palabras escuchas, en tu vocabulario diario, tu amplitud de vocabulario te afecta mucho (HC-PP-RM).

Esta posición refleja la segregación de establecimientos educacionales presente en el país. Según Carrasco y Flores (2013), en Chile las familias con altos ingresos educan a sus hijos en colegios con altos aranceles, los que a su vez se vinculan con una educación de mejor calidad. Es decir, el sistema educativo es un engranaje capaz de reproducir lenguajes de distinción entre los sujetos, replicando distinciones económico-sociales originarias (Bourdieu \& Passeron, 1979, 1990). Esto significa que el ingreso a un establecimiento educacional no es azaroso, sino producto de las condiciones sociales previas.

Por lo tanto, lo que viene a hacer la PSU es reafirmar, en términos subjetivos, la posición de privilegio de estos estudiantes: saben — son conscientes - que los conocimientos adquiridos por pertenecer a su clase social son suficientes para un buen rendimiento 
en la prueba, motivo por el cual construyen sus percepciones y expectativas sobre la base de la certidumbre. La siguiente afirmación —desde otro sentido— expresa esta situación:

nunca he sentido presión por nada, de hecho siempre he sentido apoyo por la decisión que yo quiera tomar, por ejemplo, no hubiese dado la PSU y hubiese querido estudiar repostería en el Inacap $^{26}$ (HC-PP-VIII).

Por otra parte, los estudiantes consideran que un buen desempeño en la PSU también depende de las estrategias mecánicas - que se utilizan para rendirla. Y la principal de ellas es el entrenamiento y la preparación de ensayos. Es decir, perciben la prueba como un desafío que es posible resolver mediante la repetición de ciertos ritos evaluativos.

es mucha memoria más que análisis y eso debería ser más análisis porque después, por lo que me han dicho los universitarios, el conocimiento como que después ya no te sirve tanto (HC-PP-IV).

al final es tanta práctica, tan mecánico todo según yo, espero alcanzar lo que quiero (HC-PP-RM).

es cosa de comprarte un manual y ponerte a trabajar (HC-PP-VIII).

Por lo tanto, el efecto performativo de la PSU radica en que resignifica la forma de estudiar de estos sujetos. Si la repetición, el entrenamiento y la práctica son los factores que se perciben como claves para lograr mejores resultados, entonces se movilizan distintos recursos —que en este caso son abundantes, dada su posición social_ para acomodarse a este contexto.

En relación con la valoración normativa de la PSU, predomina un ideario de justicia de tipo distributiva entre estos estudiantes. Es decir, se critica la desigual distribución de recursos educativos, así como también los posteriores resultados que se derivan de ella.

26 Instituto Nacional de Capacitación. Institución privada conformada por una universidad, instituto profesional y centros de formación técnica. 
yo creo que tengo mucha mejor formación que hartas personas y a veces eso me da lata, porque veo gente súper esforzada que le cuesta llegar a los resultados que llego yo (HC-PP-RM).

[la PSU] excluye demasiado, porque ya, nosotros acá, en este colegio, podemos pagar un preuniversitario, que además de la educación que ya recibimos, puede ayudar para subir los puntajes, pero si es que alguien no puede recibir la educación que nosotros recibimos, tampoco puede optar a un mejor puntaje. Sí, nosotros estamos muy por sobre la media de Chile (HC-PP-RM).

Asimismo, se afirma que los puntajes de la PSU evidencian un sistema educativo que no distribuye de manera equilibrada los recursos educativos de calidad.

Yo no creo que las reproduzca o que las provoque [las desigualdades], de cierta forma, las evidencia, porque no es la causa, es una manera de evidenciarlas. En la PSU se puede ver perfectamente; yo creo la segregación sociocultural en la persona de acuerdo con el nivel económico muchas veces también, todo a través del puntaje (HC-PP-RM).

Junto con esto, los estudiantes entrevistados de clase alta no solo enfatizan que la PSU se ha transformado en el elemento que permite visibilizar de manera óptima las desigualdades en el acceso a una educación básica y media, sino también que reproduce la segregación previa. Así, el ideario expuesto por estos estudiantes se vincula con una justicia distributiva, cuyo énfasis radica en evaluar diferencialmente según capacidades y necesidades.

c. Percepciones y valoraciones estudiantes entrevistados clase baja En términos generales, los estudiantes entrevistados de clase baja consideran que la PSU es un instrumento neutro y justo, por cuanto permite que todos, sin distinción de origen, puedan ser evaluados de la misma manera. Sin embargo, también perciben que para obtener un buen desempeño en la prueba es fundamental ampliar las estrategias 
de éxito, como es acceder a preuniversitarios ${ }^{27}$. En ese sentido, para ellos la desigualdad en los resultados radica principalmente en la imposibilidad de pagar por un servicio de ese tipo.

si uno no hace preuniversitario y solamente quiere llegar y dar la PSU con lo que sabe, funciona según que tanto tomaste en cuenta primero medio para solamente aprenderlo y no solamente memorizarlo para la prueba (HC-PS- RM).

hay cosas que yo no aprendí en el colegio, pero que las aprendí en el preu, por el tema de que cuando uno llega a fin de año no le alcanzan a pasar las materias por tema de los feriados y todas esas cosas, pero son las pocas, no es que en mi colegio no me hayan pasado todo (HC-PS-VI).

De hecho, en algunos casos, las escasas opciones de acceso a un preuniversitario son el factor clave para el éxito, incluso en la vida. En su acceso radica el todo o nada para el futuro.

yo soy súper memorizada, soy muy buena para todo y me faltó más apoyo económico como para hacer un preu [porque] en el colegio no te dan ese apoyo para hacer la PSU, porque el colegio te limita solamente para el lado técnico (TP-PS-IV).

Si los estudiantes perciben que para lograr un buen desempeño en la PSU deben asistir a un preuniversitario, entonces se configura nuevamente una diferencia basada en la capacidad de pago. En estos jóvenes, las expectativas se construyen esencialmente desde el factor económico. Así, lo económico rebosa todo, incluso es la razón más importante para rendir la PSU.

no, más que nada por el tema de las becas voy a dar la PSU (TPPS-VI).

En este sentido, lo económico — como figura general— se traduce en la práctica en la preocupación por el costo que significa

27 En Chile, los preuniversitarios son cursos (varían en su contenido y duración) que preparan a los estudiantes para rendir la PSU. 
cursar una carrera en ES, a diferencia de los estudiantes entrevistados de clase alta, para quienes este tema no es del todo relevante.

si no me equivoco en la universidad que yo quiero [la carrera] bordea los 4 millones 100 mil pesos y la más cara que vi 4 millones 800 mil (HC-PS-VI).

[en] Ingeniería Comercial el arancel anual son como 4 millones y medio, Auditoría eran como 5 y Arquitectura 5 y medio (TP-M-RM).

Por lo tanto, la PSU redefine el sentido que adquiere la incertidumbre en los procesos de admisión, un sentido negativo que permea el modo en que la subjetividad de los estudiantes se construye y reconstruye. A partir de ello, surgen sentimientos de miedo y angustia que afectan la forma en que enfrentan el proceso de admisión en su conjunto.

Es que lo que pasa, es que para entrar a la universidad, igual hay que tener buen puntaje y todo, entonces eso es lo que a mí me da como miedo (TP-M-VIII).

tenía muchos nervios porque de cierta forma cuando uno está ahí, como yo soy muy nervioso entonces pierdo la cabeza y respondo tonteras (TP-PS-RM).

juega en contra la presión y el nerviosismo así que aunque te vaya bien en el colegio, al estar bajo mucha presión puedes quedar en blanco (HC-PS-IV).

Estas afirmaciones confirman los hallazgos de Canales et al., (2016), quienes analizaron las trayectorias y proyecciones de los estudiantes que egresaron de cuarto año medio. Los autores concluyeron que el sentimiento de miedo es la emoción más notoria entre los estudiantes de sectores populares. Es un miedo principalmente a no ingresar a la educación superior y, por ende, a volver al trabajo simple no calificado de baja remuneración ${ }^{28}$. Los investigadores atribuyen este sentimiento a las nuevas oportunidades

$\overline{28}$ Aquí el miedo es la proyección de un fracaso frente a las oportunidades que ofrece el sistema. 
de acceso a la educación superior, que trajo mayores esperanzas de cursar estudios superiores, pero a la vez nuevas formas de exclusión, más soterradas y sutiles. El sistema de educación superior rápidamente se adaptó a esta nueva realidad —masificación de la matrícula- y segmentó por clase social. Por tanto, cursar estudios superiores, si bien genera esperanzas de movilidad social, produce simultáneamente sentimientos de frustración y resignación.

Por lo tanto, la subjetividad de los estudiantes se satura de una radical "positividad": todos pueden ingresar a la educación superior, a pesar de las pruebas de selección. Es decir, el "exceso" de oportunidades transforma las expectativas que proyectan los estudiantes frente a la educación superior: se les inculca un deseo sin límites. Ya no solo se trata de un "deber", como imperativo que influye en la toma de decisiones, sino también de "poder", como potencialidad ilimitada. Es un poder cuyo soporte es una cierta libertad de hacer lo que uno se propone, sin más barreras que la propia voluntad y el esfuerzo. Dicho de otra forma, los valores de la sociedad moderna se socializan y producen una suerte de "pensamiento unidimensional" que ahoga otras opciones de vida: al finalizar la educación secundaria no hay más alternativa que proseguir estudios superiores ${ }^{29}$. Así, los valores de la "vida activa", "culturalizada", de la "ciudadanía", se propagan, reduciéndose las opciones y proyectos de vida en el marco de aquellos discursos.

Sin embargo, este exceso de positividad ${ }^{30}$ termina por "destruir" la subjetividad del sujeto, que vacila entre el deseo de ingresar a un programa de estudio en la educación superior y el miedo a reproducir las trayectorias asociadas a su clase social. La PSU es el elemento simbólico más significativo en estos términos, puesto que es el medio a través del cual el sujeto "fracasa" en el sentido que no le es permitido

29 La subjetividad de los sujetos depende esencialmente de si ingresan o no a la educación superior. Si bien se busca el prestigio a través de la elección de ciertas carreras e instituciones, lo importante y relevante socialmente es "estar adentro", no quedar excluido de las oportunidades y experiencias formativas que "los otros" van a vivir. Se trata de obtener una "credencial", un sello que permita diferenciarse y competir por mejores empleos, e incluso para construir y reconstruir una forma de identidad.

30 La positividad, en este caso, se entiende como la constitución de espacios sin prohibición de ningún tipo. Es la posibilidad de máxima realización. 
ingresar a los espacios de privilegio o, por el contrario, se transforma definitivamente en un "sujeto de rendimiento"31 pertinente para ciertos espacios laborales de la estructura ocupacional.

Por otra parte, los estudiantes entrevistados de clase baja valoran la PSU desde una perspectiva procedimental, es decir, destacan la imparcialidad o neutralidad de su diseño. Por lo tanto, consideran que es una prueba "justa" en la medida en que evalúa a todos sobre la base de las mismas reglas. Esta perspectiva revela una idea de justicia basada en la justicia procedimental, la cual prioriza la presencia de reglas iguales para todos, independientemente de los resultados que produce.

yo creo que es justa, es súper, es que es buena, de desigualdad no po', porque todos tienen capacidades distintas. Porque unos pueden sacar mucho puntaje y otros pueden sacar poco, pero eso ya va en la capacidad que tenga uno en responder, porque una cosa es ponerle atención y responder realmente la prueba y otra es jugar al "cape nane tene tú" y responderla (TP-M-RM).

es como igualitario... porque da lo mismo de donde uno venga, si uno estudia y se prepara puede darla hasta mejor que alguien que sea de una clase social alta, entonces sí es equitativa (TP-M-RM).

En este sentido, los resultados de la PSU serían "justos" dado que todos los postulantes, independientemente de su clase social, deben cumplir los mismos requisitos y responder a las mismas preguntas. Para ellos, mientras la prueba asegure la imparcialidad, es decir, que las vacantes que ofrecen las universidades se llenen sobre la base del esfuerzo individual, entonces no hay necesidad de impugnar su existencia. Se trata, por tanto, de un argumento cuyo fundamento descansa en la idea de igualdad de oportunidades: lo fundamental no son los resultados, sino más bien garantizar que las oportunidades que se ofrecen hayan sido diseñadas sin favorecer a ningún individuo o grupo social.

31 Se menciona "sujeto de rendimiento" en el sentido que el éxito en la PSU implica no solo el ingreso a un espacio social deseado, sino también a una forma de producción de conocimientos y de trabajo particular: la autoexplotación o a la cultura del logro. Esto es la continuación de la lógica que deviene cuando se cursa un preuniversitario: lo importante es el rendimiento. Y para continuar bajo esta lógica es necesario ingresar a estos espacios sociales. 


\subsubsection{Pruebas de competencias (PC)}

\section{a. Elementos comunes}

En cuanto a las PC, en general los estudiantes valoran positivamente las premisas que sostienen su diseño, esto es, que midan competencias y habilidades que han aprendido en distintos contextos, y que no se restrinjan a aquellos conocimientos que dependen del colegio al que se asiste ${ }^{32}$. Es decir, destacan que el aprendizaje no solo se desarrolla en los espacios institucionales y formales, sino también en otros contextos, igualmente importantes, tales como el hogar, el barrio, los espacios públicos, e incluso la calle.

esa prueba igual es posible que uno se prepare personalmente para ella, por ejemplo, hay cosas de la PSU que uno tiene que buscar a alguien para que le ayude a resolverla, a ir aprendiendo cómo resolverla, y hay personas que no tienen recursos para acceder a eso, entonces considero que sí, es mejor (HC-PS-VI).

siento que la pude resolver bien, con los conocimientos que tenía del mismo colegio, porque no buscaba medir conocimiento, buscaba medir habilidades (HC-PP-RM).

es que es aplicar lo que uno conoce como de calle, como de lo que ha visto por televisión o cosas así y aplicar un poco los conocimientos que tengo de colegio finalmente, pero es más como personal, más tu idea personal (HC-PS-RM).

no sé, es que igual a uno lo hace pensar entonces igual eso sirve. En cambio, el que sea solamente de alternativas es como no una solamente es la correcta y hasta ahí no más llegaste, si te equivocaste, mala suerte, porque esa era la correcta ... en cambio con estas de desarrollo no, uno puede poner su conocimiento, su pensamiento todo ahí en la respuesta (TP-M-VI).

32 En este caso es fundamental tener presente que los estudiantes están opinando respecto de los distintos tipos de ítems de las pruebas, donde las preguntas de desarrollo resultaron la innovación más relevante. 
En alguna medida, las PC redefinen la subjetividad de los sujetos: radicalizan la idea de aprendizaje y conocimiento que de cierta manera la PSU ha diluido. En principio, con estas pruebas ya no es necesario apelar a un elemento "externo" — preuniversitario, redes, etc. - para lograr un buen desempeño. Las competencias y habilidades evaluadas se perciben como el resultado de una trayectoria que se configura por diferentes elementos, pero que al momento de la evaluación el principal factor es el desarrollo contextual y situado del conocimiento.

Por lo tanto, las PC estarían valorando de mejor forma las distintas habilidades que tienen los estudiantes. Esta situación condiciona la subjetividad en la medida que los sujetos, de alguna manera, vuelven a ser sujetos: es una suerte de "humanización" del proceso de selección. Ya no se autoperciben como "máquinas" entrenadas para responder a una prueba. Al valorar las habilidades que se desarrollan en distintos contextos pareciera haber un reordenamiento en la subjetividad que le da sentido.

Las PC no solo valoran las competencias y habilidades de una población de estudiantes cada vez más diversa, sino también las que son útiles para el mundo de hoy. Un ejemplo de esto son las preguntas de desarrollo, las que permiten mostrar habilidades antes invisibles, tales como la reflexión y el pensamiento crítico.

en el fondo estaba muy acorde a lo que se está viviendo en el siglo, que yo puedo tener información de donde quiera, puedo tener la información instantáneamente y necesito aprender, más que aprenderla de memoria, [necesito aprender] a relacionarla con lo que ya sé y a entenderla (HC-PP-RM).

yo encuentro que está bien que sea de desarrollo porque a uno lo hace pensar, lo hace reflexionar y es lo que no sé quizás lo que más sirve hoy en día que sea de alternativas que diga no solamente esta es la correcta (TP-M-VI).

En consecuencia, los estudiantes reconocen, de manera transversal, que estas pruebas son un avance, puesto que evalúan lo que es relevante para el mundo de hoy. Es decir, las PC serían más 
pertinentes que la PSU en la medida en que valoran aspectos que son esenciales no solo para cursar exitosamente un programa de estudios, sino también para un buen desempeño en el mundo laboral.

b. Percepciones y valoraciones estudiantes entrevistados clase alta Los estudiantes entrevistados de clase alta consideran que la aplicación de estas pruebas representaría un avance del sistema educacional, por cuanto son capaces de medir las "habilidades del siglo XXI", las cuales serían mucho más valiosas para la empleabilidad. Sin embargo, esbozan algunas críticas a su diseño y destacan algunas consecuencias negativas.

Por una parte, consideran que su dificultad es menor en comparación a la PSU. La impresión es que estas pruebas, si bien son valiosas, son más fáciles de responder.

porque yo creo que se me haría más fácil y no... no me confundiría porque las alternativas [de la PSU] son muy parecidas (HC-PP-IV).

Yo creo que me costaron menos, en cuanto a conocimiento y materia académica más dura (HC-PP-RM).

Debido a esta baja dificultad, los estudiantes proyectan que el proceso de selección corre el riesgo de transformarse en un simple trámite.

yo creo que sí, pero no apasionarían tanto en realidad, ahí ya pasarían a ser un trámite porque... a mí me gusta lo que es la materia académica y estudiar harto para lo que es la PSU, estudiar harto, manejarme porque tienen pillerías (HC-PP-RM).

Es decir, hay un cuestionamiento a la dificultad de la prueba por cuanto resignificaría el sentido del esfuerzo individual. El paradigma PSU, que realza el esfuerzo personal y la competencia, se articula como fuerza crítica. Para los estudiantes entrevistados de clase alta, cuyo capital cultural es mayor, una prueba de este tipo representa un desafío intelectual y cognitivo menor, y eso provoca un quiebre en su subjetividad. La necesidad de diferenciación social se expresa en esta posición. Para ellos el ingreso a la educación superior ya está 
—de alguna forma- resuelto, motivo por el cual la preocupación se desplaza hacia el rendimiento y, en particular, a la competencia que existe entre pares, es decir, entre los que pertenecen a su mismo grupo social. Las pruebas basadas en la evaluación de competencias y habilidades resignificaría esta competencia, haciéndola poco atractiva.

Frente a esto, hay una preferencia por la PSU debido a que es algo conocido y hay más certeza del rendimiento que se podría obtener.

yo daría la PSU ... porque me gusta más y es un mejor desafío (HC-PP-RM).

Entonces me gustaría la PSU, porque ya la he preparado muchísimo y sé que, en relación a la media, me va a ir bien y voy a sacar buenos puntajes porque ... si [estuviéramos] en un mundo utópico y entran todos los que pasan el puntaje de corte, me gustaría mucho más la otra prueba (HC-PP-RM).

c. Percepciones y valoraciones estudiantes entrevistados clase baja A diferencia de los estudiantes entrevistados de clase alta, los de clase baja perciben que la dificultad de las PC es mayor, a pesar de valorar la propuesta. Hay varias razones para ello, pero destaca el hecho de que, según ellos, estas pruebas miden cuestiones que han sido enseñadas tiempo atrás y que no recuerdan del todo.

creo que fue en la de Matemáticas o la de Ciencias, que preguntaron cosas de las cuales uno sabe como en octavo y no es fácil recordar desde octavo hasta cuarto medio ese tipo de materia (TP-M-IV).

igual estaba difícil, porque como ya en tercero y cuarto a mí ya no me pasan, no tengo esas materias (TP-PS-RM).

Me parecieron, sí en verdad que me parecieron más difíciles que otros ensayos que ya había hecho, es verdad que, en el preu, por ejemplo, miden la materia que te van pasando entonces van colocando más materia de la que pasaron de antes (TP-PS-RM). 
Aquí es fácil notar que los estudiantes han sido preparados -o proyectan sus estrategias de aprendizaje- de tal forma que se amparan en la memorización de contenidos. Así, la dificultad se expresa nuevamente bajo el paradigma PSU, al considerar toda evaluación como un asunto de conocimientos que se deben aprender pragmáticamente, en vez de la aplicación del mismo en diferentes contextos.

En una mirada más general, la dificultad radica en que estas pruebas evalúan habilidades analíticas, las que se encuentran distribuidas de forma más desigual en la sociedad y en el sistema escolar en particular. Al comparar, señalan que para obtener un buen resultado en la PSU basta con estudiar los últimos años y entrenar lo necesario. En el caso de las PC, para obtener un buen desempeño es necesario desarrollar habilidades durante toda la etapa escolar, cuyos resultados dependen en mayor medida del capital cultural y de la calidad de la educación recibida. Con la PSU tienen la opción de ir al "mercado de la educación" - preuniversitarios - y preparase para la prueba. En cambio, con las PC el "problema" se traslada exclusivamente al colegio y la familia. Por lo tanto, para los estudiantes entrevistados de clase baja estas pruebas son potencialmente más injustas, puesto que su diseño podría ampliar la desigualdad en los resultados dada las diferencias en los recursos culturales y educativos previos.

Sin embargo, es posible observar una fragmentación en la subjetividad de los sujetos que pertenecen a este grupo social. Esta diferencia se explica principalmente por las formas diferenciadas de exclusión que provoca la PSU. Algunos estudiantes —especialmente aquellos que cursan la modalidad técnico profesional- prefieren rendir las pruebas de competencias, si es que pudieran elegir.

[Elegiría] las pruebas piloto porque sabría a qué voy a entrar, sabría que me va a ir bien, pero habría más competencia (TPPS-IV).

yo creo que sí, es más fácil con estas porque si tuviera así desarrollo es como más, no sé más útil ... yo creo que sí porque ahí se evalúa el esfuerzo de las personas, pone de su parte en sus estudios, se evalúan amplias cosas (TP-M-VI). 
Esta preferencia radica principalmente en que estos estudiantes (TP) no tienen acceso a una parte del currículo que evalúa la PSU. Por tal motivo, una prueba que "quiebre" con aquella discriminación es recibida positivamente. Es decir, a pesar de los conflictos internos que produce esta prueba o de los riesgos que vislumbran en términos de segmentación social, estos estudiantes muestran una preferencia clara por las PC, dada la actual posición de desventaja en que se encuentran.

Por lo tanto, frente a estas percepciones de injusticia y riesgo, pero también de valoración en algunos casos, las PC redefinen -en algún grado- los sentimientos de miedo y angustia que provoca la PSU entre estos estudiantes. Si bien el miedo depende de las consecuencias de las PC en el proceso de selección — que en este caso no están claras porque se trata de un primer piloto- se constatan algunas diferencias significativas. Aquí el miedo no se traduce en angustia al fracaso, sino más bien en un asunto más particular: nerviosismo.

el nerviosismo era mucho menor, porque como te digo eran preguntas bastante más cercanas, eran más simples, bien concretas en ese sentido. Encuentro que facilitarían bastante más la equidad y el acceso a oportunidades de todos (HC-PS-VIII).

En ese sentido, cuando la prueba se percibe "más cercana" entonces es posible hablar de un cambio en la subjetividad de los sujetos. El instrumento redefine la incertidumbre y, en consecuencia, los sentimientos de angustia y temor. Sin embargo, no es plausible afirmar que se trata de una ruptura total. Así, es necesario revisar la forma y contenido de este quiebre en el contexto de un proceso formal de admisión a la educación superior.

\section{Conclusiones}

Diferencias y semejanzas en la subjetividad de los estudiantes Los hallazgos — si bien son preliminares - dan cuenta de cómo determinadas pruebas estandarizadas, utilizadas como instrumentos de selección a la educación superior, constituyen la subjetividad 
de los estudiantes en términos de percepciones y valoraciones normativas. O dicho de otra forma, el estudio indica la forma en que la subjetividad de los estudiantes que finalizan la educación secundaria deviene en relación con la PSU y las nuevas PC diseñadas por el DEMRE. El análisis se centró en identificar algunas diferencias y semejanzas según la posición social de los estudiantes. Al respecto, se encontraron ciertos patrones en función de la clase social que permiten establecer algunas distinciones entre ellas, pero también se evidenciaron contradicciones internas que muestran el carácter fragmentario y conflictivo de las subjetividades situadas socialmente.

En relación con los instrumentos de evaluación, a pesar de las críticas que recibe de algunos estudiantes, la PSU se concibe como parte constituyente del proceso de elección. Hay una valoración positiva transversal, puesto que contiene una promesa: el desempeño depende esencialmente del esfuerzo. En otros términos, la PSU refleja el paradigma meritocrático en el sistema de educación superior, motivo por el cual su existencia asegura que los resultados de los procesos de selección son justos. Los cuestionamientos no se centran en su existencia, sino más bien en el contexto de desigualdad donde se inserta, en términos de la calidad y pertinencia de los recursos educativos disponibles en los distintos grupos sociales.

Al observar la valoración de la PSU según la posición social de los estudiantes, se aprecian algunas diferencias. Los estudiantes entrevistados pertenecientes a la clase alta consideran que la prueba es un instrumento limitado en cuanto a su capacidad para definir quiénes son aptos para ingresar a la educación superior, puesto que excluye a potenciales talentos ocultos. Junto con esto, no solo enfatizan que la PSU se ha transformado en el elemento que permite visibilizar las desigualdades en el logro académico, sino también que reproduce y amplifica la segregación previa. Por tanto, el ideario expuesto por estos estudiantes se vincula principalmente con una justicia distributiva, dado que enfatizan las diferencias en los recursos educativos y la reproducción de dichas brechas.

Por su parte, los estudiantes entrevistados de clase baja, en general consideran que la PSU es un instrumento neutro y justo, por 
cuanto permite que todos, sin distinción de origen, sean evaluados de la misma manera. Junto con ello, identifican una fuerte conexión entre mejores resultados PSU y el acceso a preuniversitarios, sosteniendo que la desigualdad reside en las (im)posibilidades de pagar un servicio de ese tipo. Esta perspectiva releva una justicia procedimental, la cual prioriza y valora la imparcialidad de la prueba misma, es decir, la presencia de reglas iguales para todos.

Respecto de las PC, en general los estudiantes valoran positivamente que midan lo que han aprendido en el colegio. Los estudiantes de sectores acomodados consideran que la aplicación de estas pruebas representaría un avance del sistema educacional por cuanto son capaces de medir las "habilidades del siglo XXI", las cuales serían mucho más valiosas para un buen desempeño en la educación superior y en el mercado laboral. Sin embargo, para algunos estudiantes entrevistados que pertenecen a la clase baja, estas pruebas son potencialmente más injustas que la PSU, puesto que evalúan habilidades analíticas, las que se encuentran distribuidas de forma más desigual en la sociedad y en el sistema escolar en particular. Al comparar, señalan que para obtener un buen resultado en la PSU basta con estudiar los últimos años y entrenar. En cambio, para obtener un buen desempeño en las PC es necesario desarrollar habilidades durante toda la etapa escolar, cuestión que depende en mayor medida del capital cultural y de la calidad de la educación escolar recibida. Con la PSU tienen la opción de ir al "mercado de la educación" (preuniversitarios) y preparase estratégicamente. En cambio, con las PC el "problema" descansa en el proceso de enseñanza que imparten los colegios, así como también en la familia. Para los estudiantes provenientes de la formación TP, si bien comparten estos reparos, prefieren las PC en vez de la PSU porque las perciben mucho más cercanas en términos de los contenidos que contemplan y el tipo de ítems que se utilizan. Es decir, se aprecia una diferencia entre los estudiantes TP y los PS en relación con las nuevas PC que se explica principalmente por la "lucha" que hay por las mismas oportunidades en función de la posición social que comparten: la competencia por una vacante no es con los estudiantes pertenecientes a la clase alta, sino con aquellos más cercanos socialmente. 


\section{Perspectivas futuras}

El objetivo de las PC es ampliar las oportunidades de ingreso a la educación superior a todos los estudiantes de educación secundaria, independientemente de la modalidad que hayan cursado, a través de la evaluación de competencias fundamentales y habilidades transversales. Estas competencias refieren a aspectos esenciales del currículo nacional y se vinculan a aquellas necesarias para cumplir satisfactoriamente un programa de estudios en el nivel terciario.

Por lo tanto, la necesidad de diseñar instrumentos de evaluación que no generen ventajas ni desventajas a subgrupos particulares de estudiantes es un elemento fundamental para cumplir con el criterio técnico de imparcialidad que plantean los estándares internacionales (American Educational Research Association, AERA, American Psychological Association, APA, \& National Council on Measurement in Education, NCME, 2014). Sin embargo, los resultados del estudio muestran que estos criterios son cuestionados por los estudiantes, al menos por un grupo de ellos.

El principal cuestionamiento radica en que introducen un elemento de injusticia en la medida en que evalúan un aspecto cuya distribución en la sociedad es más desigual. Por lo tanto, se evidencia una tensión entre los objetivos de las PC y el sentido social que los estudiantes le atribuyen. Esta situación merece atención, puesto que no solo implica reflexionar en torno al diseño del instrumento, sino también en su recepción en el conjunto del sistema, lo que incluye a profesores, estudiantes y padres.

A partir de estos resultados emanan algunas reflexiones, así como también nuevas interrogantes. Respecto de lo primero, es relevante considerar las consecuencias que produce la evaluación de competencias en el sistema de educación en su conjunto. Las modificaciones en los sistemas de admisión a la educación superior ciertamente producen cambios en los procesos formativos. Los colegios generan estrategias para adaptarse a los nuevos requerimientos, y algunos están más preparados para ello que otros. Es decir, si asumimos la tesis de que las clases sociales, principalmente las que se encuentran en posiciones privilegiadas, buscan mantener su posición 
y reproducirla, entonces es plausible concluir que los colegios más aventajados —en términos de capital económico y cultural — serán los primeros en favorecer y promover este tipo de formación. Así, las brechas sociales se mantienen a lo largo del tiempo.

Adicionalmente, un estudio complementario (Lavados, Giaconi, Jiménez y Osses, 2018) muestra que la brecha de resultados entre estudiantes provenientes de la modalidad HC y TP disminuye en las PC, en comparación con el desempeño en la PSU de Lenguaje y Matemática. Esto contradice de alguna manera las percepciones de los estudiantes antes mencionados.

Al respecto, surge la pregunta acerca del contenido de esa diferencia, más allá de su disminución. Es decir, no hay claridad si se trata de un desplazamiento "hacia abajo" de los que habitualmente han tenido mejor desempeño, o bien hay un desplazamiento "hacia arriba" de quienes tradicionalmente reportan desempeños insuficientes. Conocer y comprender la forma, condiciones y contenido de esa brecha emerge necesariamente como una nueva interrogante a indagar.

\section{Referencias}

American Educational Research Association, AERA, American Psychological Association, APA, \& National Council on Measurement in Education, NCME. (2014). Standards for educational and psychological testing. Washington, D.C: American Educational Research Association.

Ball, S. J. (2003). The teacher's soul and the terrors of performativity. Journal of Education Policy, 18(2), 215-228. https://doi.org/10.1080/0268093 022000043065

Ball, S. J. (2012). Performativity, commodification and commitment: An I-Spy guide to the neoliberal university. British Journal of Educational Studies, 60(1), 17-28. https://doi.org/10.1080/00071005.2011.650940

Ball, S. J. (2015). Living the neo-liberal university: Living the neo-liberal university. European Journal of Education, 50(3), 258-261. https://doi. org/10.1111/ejed.12132

Ball, S. J. (2017). Foucault as educator. Recuperado de //www.springer.com/ la/book/9783319503004 
Ball, S. J. \& Olmedo, A. (2013). Care of the self, resistance and subjectivity under neoliberal governmentalities. Critical Studies in Education, 54(1), 85-96. https://doi.org/10.1080/17508487.2013.740678

Barnett, R. \& Di Napoli, R. (Eds.). (2008). Changing identities in higher education: Voicing perspectives. Londres: Routledge.

Barozet, E. y Mac-Clure, O. (2015). Tolerancia a la desigualdad y justicia social. Una agenda teórica de investigación. En M. Castillo y C. Maldonado (Eds.), Desigualdades. Tolerancia, legitimación y conflicto en las sociedades latinoamericanas (pp. 151-182). Santiago de Chile: RIL Editores.

Bourdieu, P. \& Passeron, J. C. (1979). The inheritors: French students and their relation to culture. Chicago: University of Chicago Press.

Bourdieu, P. \& Passeron, J. C. (1990). Reproduction in education, society, and culture. Londres; Newbury Park, Calif: Sage in association with Theory, Culture \& Society, Dept. of Administrative and Social Studies, Teesside Polytechnic.

Burrows, R. (2012). Living with the H-Index? Metric assemblages in the contemporary academy. The Sociological Review, 60(2), 355-372. https://doi.org/10.1111/j.1467-954X.2012.02077.x

Callon, M. (2007). What does it mean to say that economics is performative? En D. MacKenzie, F. Muniesa, \& L. Siu (Eds.), Do economics make markets? On the performativity of economics (pp. 311-357). Princeton, New Jersey: Princeton University Press.

Callon, M. (2010). Performativity, misfires and politics. Journal of Cultural Economy, 3(2), 163-169. https://doi.org/10.1080/17530350.2010.4 94119

Canales, M., Ghiardo, F., y Opazo, A. (2015). Para un concepto de juventud. En P. Cottet (Ed.), Juventudes: metáforas del Chile contemporáneo. Santiago de Chile: RIL Editores.

Canales, M., Opazo, A., y Camps, J. P. (2016). Salir del cuarto: expectativas juveniles en el Chile de hoy. Última Década, 24(44), 73-108. https:// doi.org/10.4067/S0718-22362016000100004

Carrasco, A. y Flores, C. (2013). (Des)igualdad de oportunidades para elegir escuela: preferencias, libertad de elección y segregación escolar. Santiago de Chile: Espacio Público. Recuperado de https://www.researchgate.net/ publication/318471484_Desigualdad_de_oportunidades_para_elegir_ escuelas_Preferencias_libertad_de_eleccion_y_segregacion_escolar

Dean, M. (2010). Governmentality: Power and rule in modern society (2a ed.). Londres: Thousand Oaks, Calif: SAGE. 
Dubet, F. (2012). Repensar la justicia social: contra el mito de la igualdad de oportunidades. Buenos Aires: Siglo XXI Editores.

Foucault, M. (1982). The subject and power. Critical Inquiry, 8(4), 777-795. https://doi.org/10.1086/448181

Foucault, M. (2009). Security, territory, population: Lectures at the Collège de France 1977-78. Basingstoke: Palgrave Macmillan.

Foucault, M. (2010a). The birth of biopolitics: Lectures at the Collège de France, 1978-79. (M. Senellart, Ed., G. Burchell, Trans.). Nueva York, NY: Palgrave Macmillan.

Foucault, M. (2010b). The government of self and others. Houndmills, Basingstoke, Hampshire, Nueva York: Palgrave Macmillan; St Martin's Press.

Lavados, J., Giaconi, V., Jiménez, D., y Osses, A. (2018). Brechas en el acceso a la educación superior: evaluando competencias. Santiago de Chile: DEMRE, Universidad de Chile.

MacKenzie, D. A., Muniesa, F., \& Siu, L. (Eds.). (2008). Do economists make markets? On the performativity of economics. Princeton, NJ Oxford: Princeton University Press.

Marshall, T. H. (1973). Class, citizenship, and social development: Essays. Westport, Conn: Greenwood Press.

Méndez, M. L. \& Gayo, M. (2019). Upper middle class social reproduction. Cham: Springer International Publishing. https://doi.org/10.1007/9783-319-89695-3

Ministerio de Educación, Mineduc (2016). Mensaje de S.E. la Presidenta de la República con el que inicia un proyecto de Ley de Educación Superior, Pub. L. No Mensaje $N^{\circ} 110-364$.

Neary, M. \& Winn, J. (2016). Against academic identity. Higher Education Research \& Development, 35(2), 409-412. https://doi.org/10.1080/07 294360.2015.1094201

Orellana, V. (2011). Nuevos estudiantes y tendencias emergentes en educación superior. Una mirada al Chile del mañana. En M. Jiménez y F. Lagos (Eds.), Nueva geografía de la educación superior. Santiago de Chile: Foro Aequalis.

Orellana, V., Guzmán, C., Bellei, C., Gareca, B., y Torres, F. (2017). Elección de carrera y universidad en Chile: sentido y utilidad de la acreditación. Cuadernos de investigación en aseguramiento de la calidad No. 7. Santiago de Chile: Comisión Nacional de Acreditación. 
Peters, M. \& Burbules, N. C. (2004). Poststructuralism and educational research. Lanham, Md: Rowman \& Littlefield Publishers.

Ramos, C. (2012). El ensamblaje de ciencia social y sociedad: conocimiento científico, gobierno de las conductas y producción de lo social. Santiago de Chile: Ediciones Universidad Alberto Hurtado. Recuperado de http:// repositorio.uahurtado.cl:80/handle/11242/4917

Rawls, J. (2005). A theory of justice. Cambridge, Mass: Belknap Press.

Rose, N. S. (2005). Governing the soul: The shaping of the private self (2. ed., [reprint]). Londres: Free Association Books.

Sutton, P. (2017). Lost souls? The demoralization of academic labour in the measured university. Higher Education Research \& Development, 36(3), 625-636. https://doi.org/10.1080/07294360.2017.1289365

Torres, R. y Zenteno, M. E. (2011). El sistema de educación superior: una mirada desde las instituciones y sus características. En M. Jiménez y F. Lagos (Eds.), Nueva geografía de la educación superior (pp. 13-77). Santiago de Chile: Foro Aequalis.

Turner, J. H. (2007). Justice and emotions. Social Justice Research, 20(3), 288311. https://doi.org/10.1007/s11211-007-0043-y

Zima, P. V. (2015). Subjectivity and identity: Between modernity and postmodernity. Londres; Nueva York: Bloomsbury Academic.

Recibido: 30/07/2018

Aceptado: 05/03/2019 